\section{Noninvasive assessment of breast cancer risk using time-resolved diffuse optical spectroscopy}

\author{
Paola Taroni, ${ }^{a, b, *}$ Antonio Pifferi, ${ }^{a, b}$ Giovanna Quarto, ${ }^{a}$ \\ Lorenzo Spinelli, ${ }^{b}$ Alessandro Torricelli, ${ }^{a}$ \\ Francesca Abbate, ${ }^{\mathrm{c}}$ Anna Villa, ${ }^{\mathrm{c}}$ Nicola Balestreri, ${ }^{\mathrm{d}}$ \\ Simona Menna, ${ }^{\mathrm{c}}$ Enrico Cassano, ${ }^{\mathrm{c}}$ and Rinaldo Cubeddu, ${ }^{\mathrm{a}, \mathrm{b}}$ \\ a Politecnico di Milano, Dipartimento di Fisica, Piazza Leonardo da \\ Vinci 32, 20133 Milano, Italy \\ ${ }^{\mathrm{b}}$ Consiglio Nazionale delle Ricerche, Istituto di Fotonica e \\ Nanotecnologie, Piazza Leonardo da Vinci 32, 20133 Milano, Italy \\ CEuropean Institute of Oncology, Breast Imaging Unit, \\ Via G. Ripamonti, 435, 20141 Milano, Italy \\ dEuropean Institute of Oncology, Department of Radiology, \\ Via G. Ripamonti, 435, 20141 Milano, Italy
}

\begin{abstract}
Breast density is a recognized strong and independent risk factor for breast cancer. We propose the use of time-resolved transmittance spectroscopy to estimate breast tissue density and potentially provide even more direct information on breast cancer risk. Time-resolved optical mammography at seven wavelengths (635 to $1060 \mathrm{~nm}$ ) is performed on 49 subjects. Average information on breast tissue of each subject is obtained on oxy- and deoxyhemoglobin, water, lipids, and collagen content, as well as scattering amplitude and power. All parameters, except for blood volume and oxygenation, correlate with mammographic breast density, even if not to the same extent. A synthetic optical index proves to be quite effective in separating different breast density categories. Finally, the estimate of collagen content as a more direct means for the assessment of breast cancer risk is discussed. ( 2010 Society of Photo-Optical Instrumentation Engineers. [DOI: 10.1117/1.3506043]
\end{abstract}

Keywords: breast density; absorption; scattering; tissue spectroscopy; breast cancer.

Paper 10438LR received Aug. 6, 2010; revised manuscript received Sep. 23, 2010; accepted for publication Oct. 1, 2010; published online Dec. 2, 2010

Breast cancer is a leading cause of death in women and a major health burden worldwide. ${ }^{1}$ Breast density is a recognized strong and independent risk factor for breast cancer. ${ }^{2}$ Including it into risk prediction models improves their prediction accuracy. The United States Preventive Services Task Force also suggested the possibility of chemoprevention for women at high risk. ${ }^{3}$ Thus, improved risk models could be used to better address closer screening of high-risk women, and even for prevention of breast cancer.

At present, breast density is assessed based on the radiological appearance of breast tissue. A means for its noninvasive estimate would certainly be of diagnostic interest. Optical tech-

\footnotetext{
*Address all correspondence to: Paola Taroni, Politecnico di Milano, Dipartimento di Fisica, Piazza Leonardo da Vinci 32, Milano, 20133 Italy. Tel: 3902 23 996109; Fax: 3902 23996126; E-mail: paola.taroni@fisi.polimi.it.
}

niques can provide functional and structural information in an absolutely noninvasive way, and they have already been applied successfully to the characterization of breast tissue. ${ }^{4-6}$ Specific effort was devoted to optical estimates of breast density. In particular, scattering features proved to correlate with it. ${ }^{7,8}$ Also, extensive clinical trials showed that optical data interpreted using principal component analysis strongly correlate with quantitative mammographic features. ${ }^{9}$ A study on the direct correlation of breast density with optically derived key breast constituents (water, lipids, collagen, and hemoglobin) could lead to a better understanding of the role of mammographic density in breast cancer, and possibly provide a more specific link. Such a study is still needed.

We propose the use of time-resolved transmittance spectroscopy to estimate breast tissue density and potentially provide even more direct information on breast cancer risk.

Our portable clinical instrument for time-resolved optical mammography operates in transmittance geometry on the mildly compressed breast. ${ }^{10}$ Time-resolved transmittance data are collected at seven wavelengths in the range 635 to $1060 \mathrm{~nm}$, using picosecond pulsed diode lasers as light sources, and two photomultiplier tubes and PC boards for time-correlated single photon counting to detect the time distributions of the transmitted pulses. Injection and collection fibers are scanned in tandem over the compressed breast, and data are stored every millimeter. Images are routinely acquired from both breasts in cranio-caudal (CC) and oblique (OB, $45 \mathrm{deg})$ views. Time-resolved spectral data are interpreted with a spectrally constrained global fitting procedure to estimate tissue composition in terms of oxy- and deoxyhemoglobin, water, lipid, and collagen content, as well as scattering parameters (amplitude $a$ and power $b$ ). For breast lesion detection, scattering maps are routinely applied, together with late gated intensity images that are sensitive to spatial changes in the absorption properties.

The instrument is presently applied in a clinical study approved by the Institutional Review Board of the European Institute of Oncology with a two-fold aim: the optical characterization of malignant and benign breast lesions, and the noninvasive assessment of breast density. The present work focuses on the latter aim. Thus, for each patient all data (from $\mathrm{CC}$ and $\mathrm{OB}$ views of both breasts) were averaged to provide the average optical properties and breast tissue composition of that subject. Data were collected from 49 patients (age $51.4 \pm 11.5$ years, body mass index $23.3 \pm 3.9 \mathrm{~m}^{2} / \mathrm{kg}$ ) recruited between June 2009 and March 2010. Written informed consent was obtained from all patients. For four patients, recent $x$-ray mammograms were not available, thus they were excluded from further analysis. An expert radiologist assigned breast imaging and reporting data system (BI-RADS) mammographic density categories as: 1, almost entirely fat $(n=6) ; 2$, scattered fibroglandular densities $(n=19) ; 3$, heterogeneously dense $(n=14)$; and 4 extremely dense $(n=6)$.

Tissue composition, as derived from optical data, and scattering parameters were estimated to investigate if dependence on mammographic density is observed. The results are summarized in Fig. 1. Water content increases progressively and significantly with breast density. Correspondingly, a gradual decrease is observed in lipid content. The amount of collagen also increases

1083-3668/2010/15(6)/060501/3/\$25.00 (C) 2010 SPIE 


\section{JBO LETTERS}
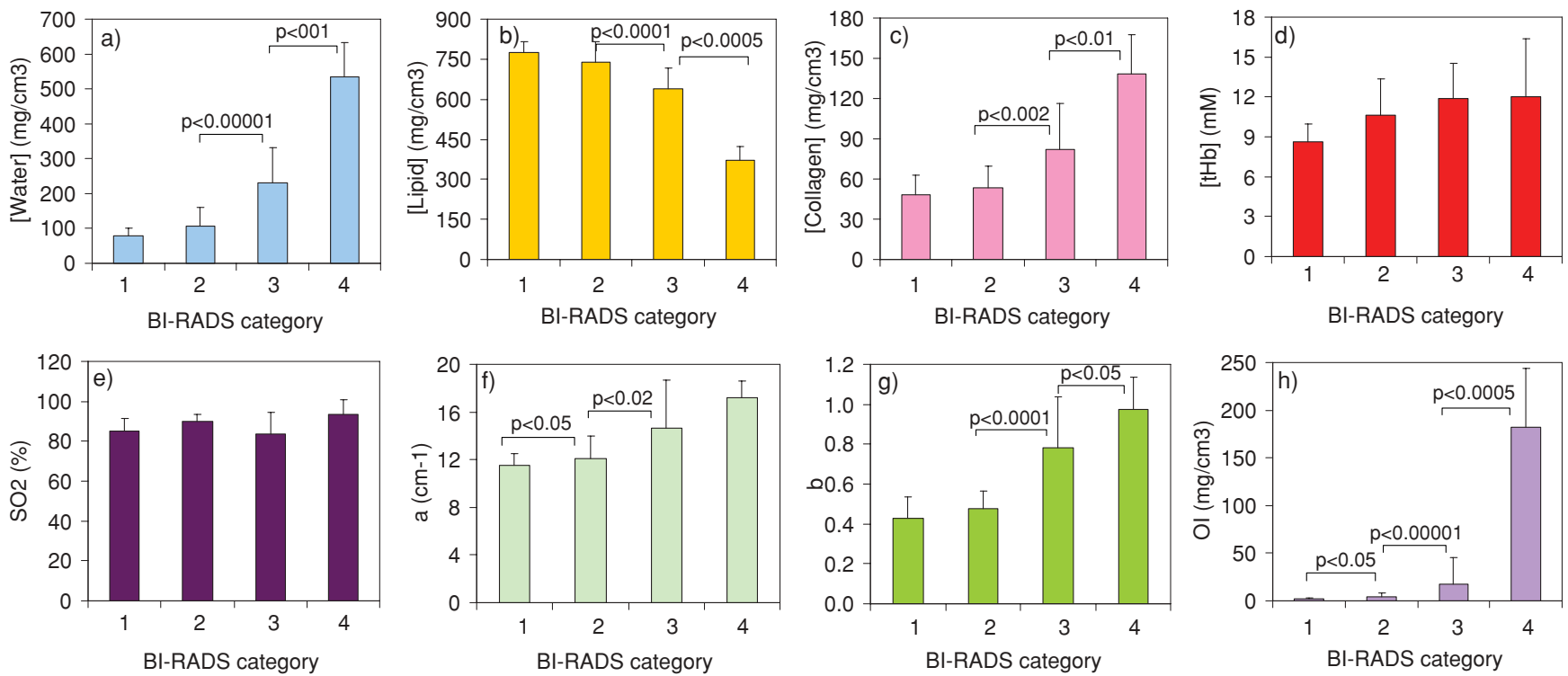

Fig. 1 Optically derived parameters versus BI-RADS categories: (a) water, (b) lipid, (c) collagen, (d) tHb, (e) SO 2 , (f) a, (g) $b$, and (h) OI. Results are expressed as mean \pm SD and refer to the intersubject variability. Statistical significance of the difference between categories was estimated with the Mann-Whitney test. $p$ values $<0.05$ are considered significant and reported in the figure.

for increasing breast density. For all three main constituents, the difference between categories is always highly significant, except between category 1 (fatty) and 2 (scattered). It is worth noting that for water, the $p$ value is very close to significance even between categories 1 and $2(p=0.0523)$. Those trends strongly agree with those expected, based on the definition of BI-RADS categories. Collagen is associated to fibroglandular tissue and thus anticipated to be present at higher concentration in dense breasts. ${ }^{11}$ However, the relative abundance of collagen in breast tissue is low, while water and lipid account for most of the breast volume, and radio-opacity is predominantly due to water, thus explaining the stronger correlation of BI-RADS categories with water and lipid amounts than with collagen content.

As compared to adipose tissue, high-density tissue can be expected to have increased vascularization and metabolism. Based on optical data, higher total hemoglobin content (tHb) seems to increase slightly with increasing density, even though in general the changes are not statistically significant. The oxygenation level $\left(\mathrm{SO}_{2}\right)$ reveals no trend at all with breast density.

The scattering parameters were also considered, as they provide information on the microscopic tissue structure that is at the origin of breast density. Both $a$ and $b$ (Fig. 1) show a similar behavior to collagen: a progressive increase is observed for increasing density categories, but it is not always statistically significant. A steeper scattering slope typically characterizes fibroglandular and muscular tissues as compared to adipose tissue. ${ }^{12,13}$ Performing measurements ex vivo on different types of bovine tissue, we have recently observed a potential correlation between scattering slope and collagen content, with ligament showing by far both the steepest slope and the highest collagen content. ${ }^{14}$ This trend is likely due to the presence of collagen fibrils with submicrometer diameter that introduce high power scattering components. Due to the different slope of the scattering spectra of distinct tissues, the amplitude $a$ (i.e., the scattering coefficient at $600 \mathrm{~nm}^{10}$ ) is expected and often observed to be lower in more adipose tissues.
In summary, consistent with breast physiology, several figures proved to correlate with mammographic breast density: water, lipid, and collagen content, as well as the scattering parameters. Their contributions may not be fully independent. Still, their combination could result in improved correlation with mammographic density. To test this hypothesis, in line with what was previously proposed by other researchers to optimize the diagnostic value of optical data, ${ }^{15,16}$ we considered an index based on optically derived parameters, defined as followed:

$$
O I=\frac{[\text { water }][\text { collagen }] b}{[\text { lipid }]}
$$

where constituent concentrations appear in square brackets. It is a very rough preliminary way to combine all relevant parameters, still, overall it improves the significance of the correlation between optical data and mammographic density, as shown in Fig. 1.

The scattering amplitude $a$ also shows some positive correlation with breast density. If included in the optical index $O I$ as a multiplying factor, it has limited effect. Specifically, it slightly degrades the performance in the discrimination of subjects at highest risk ( $p<0.001$ instead of $p<0.0005$ between categories 3 and 4). Since the identification of subjects in category 4 is of high interest for risk assessment, $a$ was excluded from the index. However, it will be further checked when data are available on a higher number of subjects. The results obtained using $O I$ are already quite effective, but we still will investigate other more refined statistical approaches (e.g., cluster analysis) for the separation of breast density classes and estimates of predictive values.

Mammographic density correlates with cancer risk. Thus, as mentioned before, it would be valuable to identify a parameter that correlates with it and can be measured noninvasively. However, it is also worth noting that mammographic density might provide only an indirect correlation with risk. X-rays are mostly sensitive to the water content, which increases with the fibroglandular component in breast tissue. However, collagen 
is perhaps the most important extracellular matrix protein in determining stromal architecture. Alterations of stromal architecture and composition are a well-known aspect of both benign and malignant pathologies, and can play an initial role in breast carcinogenesis. ${ }^{11}$ Studies performed on mice also showed that increased collagen density increases tumorigenesis, local invasion, and metastatic character, proving a causal link between stromal collagen and tumor formation and progression. ${ }^{17}$ Thus, the noninvasive estimate of collagen content in breast by optical means, exploiting the unique spectral features of collagen absorption, could provide a powerful piece of information for risk assessment, and deserves further investigation.

In summary, we show the direct correlation between mammographic density and tissue composition, in terms of all the main constituents (i.e., water, lipid, and collagen), as estimated from optical data. Clinical work is still in progress to collect data from a wider population. This will allow us to confirm the correlation between optical parameters and mammographic density, and to better investigate the potential role of collagen in the assessment of breast cancer risk.

\section{References}

1. F. Bray, P. McCarron, and D. M. Parkin, "The changing global patterns of female breast cancer incidence and mortality," Breast Cancer Res. 6, 229-239 (2004).

2. V. A. McCormack and I. dos Santos Silva, "Breast density and parenchymal patterns as markers of breast cancer risk: a meta-analysis," Cancer Epidemiol. Biomarkers Prev. 15, 1159-1169 (2006).

3. U.S. Preventive Services Task Force, "Chemoprevention of breast cancer: recommendations and rationale," Ann. Intern. Med. 137, 56-58 (2002).

4. P. Taroni, A. Bassi, D. Comelli, A. Farina, R. Cubeddu, and A. Pifferi, "Diffuse optical spectroscopy of breast extended to $1100 \mathrm{~nm}, " J$. Biomed. Opt. 14, 054030 (2009).

5. N. Shah, A. E. Cerussi, D. Jakubowsky, D. Hsiang, J. Butler, and B. J. Tromberg, "Spatial variations in optical and physiological properties of healthy breast tissue," J. Biomed. Opt. 9, 534-540 (2004).

6. B. W. Pogue, S. Jiang, H. Dehghani, C. Kogel, S. Soho, S. Srinivasan, X. Song, S. P. Poplack, and K. D. Paulsen, "Characterization of hemoglobin, water and NIR scattering in breast tissue: analysis of intersubject variability and menstrual cycle changes relative to lesions," $J$. Biomed. Opt. 9(3), 541-552 (2004).

7. X. Wang, B. W. Pogue, S. Jiang, X. Song, K. D. Paulsen, C. Kogel, S. P. Poplack, and W. A. Wells, "Approximation of Mie scattering parameters in near-infrared tomography of normal breast tissue in vivo," J. Biomed. Opt. 10(5), 051704 (2005).

8. N. Shah, A. E. Cerussi, D. Jakubowski, D. Hsiang, J. Butler, and B. J. Tromberg, "The role of diffuse optical spectroscopy in the clinical management of breast cancer," Disease Markers 19, 95-105 (2003).

9. K. M. Blackmore, J. A. Knight, and L. Lilge, "Association between transillumination breast spectroscopy and quantitative mammographic features of the breast," Cancer Epidemiol. Biomarkers Prev. 17, 10431050 (2008)

10. P. Taroni, A. Pifferi, E. Salvagnini, L. Spinelli, A. Torricelli, and R. Cubeddu, "Seven-wavelength time-resolved optical mammography extending beyond $1000 \mathrm{~nm}$ for breast collagen quantification," Opt. Expr. 17, 15932-15946 (2009).

11. Y.-P. Guo, L. J. Martin, W. Hanna, D. Banerjee, N. Miller, E. Fishell, R. Khokha, and N. F. Boyd, "Growth factors and stromal matrix proteins associated with mammographic densities," Cancer Epidemiol. Biomarkers Prev. 10, 243-248 (2001).

12. V. G. Peters, D. R. Wyman, M. S. Patterson, and G. L. Frank, "Optical properties of normal and diseased breast tissues in the visible and near infrared," Phys. Med. Biol. 35, 1317-1334 (1990).

13. M. Bartek, X. Wang, W. Wells, K.D. Paulsen, and B.W. Pogue, "Estimation of subcellular particle size histograms with electron microscopy for prediction of optical scattering in breast tissue," J. Biomed. Opt. 11, 064007 (2006).

14. P. Taroni, A. Bassi, A. Farina, R. Cubeddu, and A. Pifferi, "Role of collagen scattering for in vivo tissue characterization title of paper," in Biomedical Optics (BIOMED) on CD ROM, BTuD107, The Optical Society, Washington, DC (2010).

15. A. Cerussi, N. Shah, D. Hsiang, A. Durkin, J. Butler, and B. J. Tromberg, "In vivo absorption, scattering, and physiologic properties of $58 \mathrm{ma}-$ lignant breast tumors determined by broadband diffuse optical spectroscopy," J. Biomed. Opt. 11, 044005 (2006).

16. S. D. Konecky, R. Choe, A. Corlu, K. Lee, R. Wiener, S. M. Srinivas, J. R. Saffer, R. Freifelder, J. S. Karp, N. Hajjioui, F. Azar, and A. G. Yodh, "Comparison of diffuse optical tomography of human breast with whole-body and breast-only positron emission tomography," Med. Phys. 35, 446-455 (2008)

17. P. P. Provenzano, D. R. Inman, K. W. Eliceiri, J. G. Knittel, L. Yan, C. T. Rueden, J. G. White, and P. J. Keely, "Collagen density promotes mammary tumor initiation and progression," BMC Med. 6, 11 (2008). 